\title{
Non-Hodgkin Lymphoma of Hard Palate
}

\author{
Akshada Vithal Dhuma , Haritosh K Velankar*, Cassandra Carvalho, Stuti Mathur and Bhagyashree Sagane \\ Department of Otolaryngology, D Y Patil University, India
}

*Corresponding author: Haritosh K Velankar, Department of Otolaryngology, DY Patil University, India

\begin{abstract}
Oral cavity lesions are common complaints in clinical ENT settings. They are often misdiagnosed as a periodontal disease or granulomas. A 43-year-old male came to the our OPD with complaints of hard palate swelling on the right side for 1 year coupled with a swelling on the floor of the mouth for 2 years.
\end{abstract}

Keywords: Non-Hodgkin Lymphoma; Lymphoma; Hard Palate; Tumor; Maxilla

\section{Introduction}

Lymphomas are primarily tumours of lymph nodes. They are further classified as Hodgkin lymphoma and Non-Hodgkin lymphoma. Non-Hodgkin lymphoma is primarily present in Lymph nodes. Its extra nodal presentation includes sites like GI tract, Waldeyer's ring, lung, liver, spleen, bone, skin. However, it is rare to find Non-Hodgkin lymphomas in oral cavity and are thus often misdiagnosed. Vigilant clinical examination, radiological investigations and histopathological reports help in identifying the disease at an early stage. We therefore emphasize on a voracious workup to hasten diagnosis and early treatment. We report a rare case of a 43-year-old male presenting with a hard palate swelling (Figure 1). The swelling was associated with pain during chewing. His contrast enhanced CT face/neck was suggestive of soft tissue mass involving right half of hard palate up to the midline with bony erosion of the floor of maxillary sinus. Excision of growth was done and sent for Histopathology. Histopathology report of this growth showed Follicular B cell lymphoma.

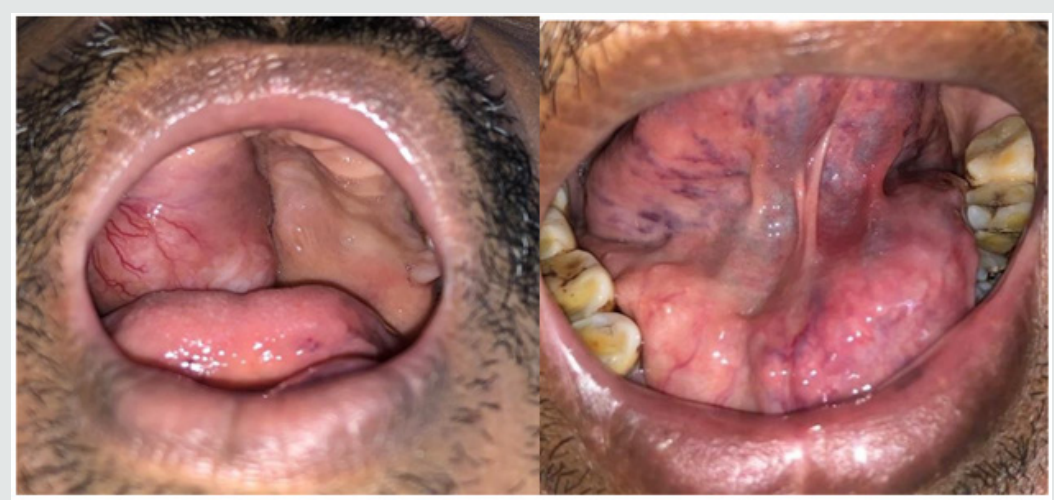

Figure 1.

\section{Case Report}

A 43-year-old male presented with complaints of swelling over the right half of the hard palate for 2 years along with swelling over the floor of the mouth on the left side for 1 year. He gave history of tobacco chewing for 15 years along with significant weight loss. The patient presented to us 2 years back when the lesion initially presented, failing to follow up. Examination: On intraoral inspection a $6 * 4 * 4 \mathrm{~cm}$ lesion on the right side of hard palate extending from upper right canine to the third molar, crossing the midline. The swelling was firm, with visible dilated veins, without scars, sinuses or pulsations. On palpation it was cystic to firm in consistency, smooth, without any local rise of temperature. On inspection 
an ovoid, solitary swelling on the left side of floor of the mouth, measuring $5.5^{*} 3^{*} 3 \mathrm{~cm}$ was noted which occluded the gingivolabial sulcus without signs of scars, sinuses, discharge from the swelling. The swelling was firm and non-tender. Indirect laryngoscopy examination was normal.

\section{Investigations}

A Face CT along with that of paranasal sinuses was ordered so as to estimate the extent of the swelling which was suggestive of a soft tissue lesion involving the right hard palate, upper alveolus and bony erosion of the floor of the maxillary sinus. The swelling also extended posteriorly up to the right superior retromolar trigone causing erosion of the right medial pterygoid plate. The scan also revealed multiple non necrotic cervical lymph nodes on bilateral level Ib, II and Va. Following this an excision biopsy was planned for further evaluation (Figure 2). The samples were sent for histopathological evaluation. Biopsy revealed Lymphoid tissue arranged in variable sizes separated by thin and thick fibro cartilagenous septae was seen suggestive of? Non-Hodgkin lymphoma. For definitive diagnosis and to measure the extent/ spread of the condition FNAC of the cervical lymph nodes and Bone marrow aspiration was performed.

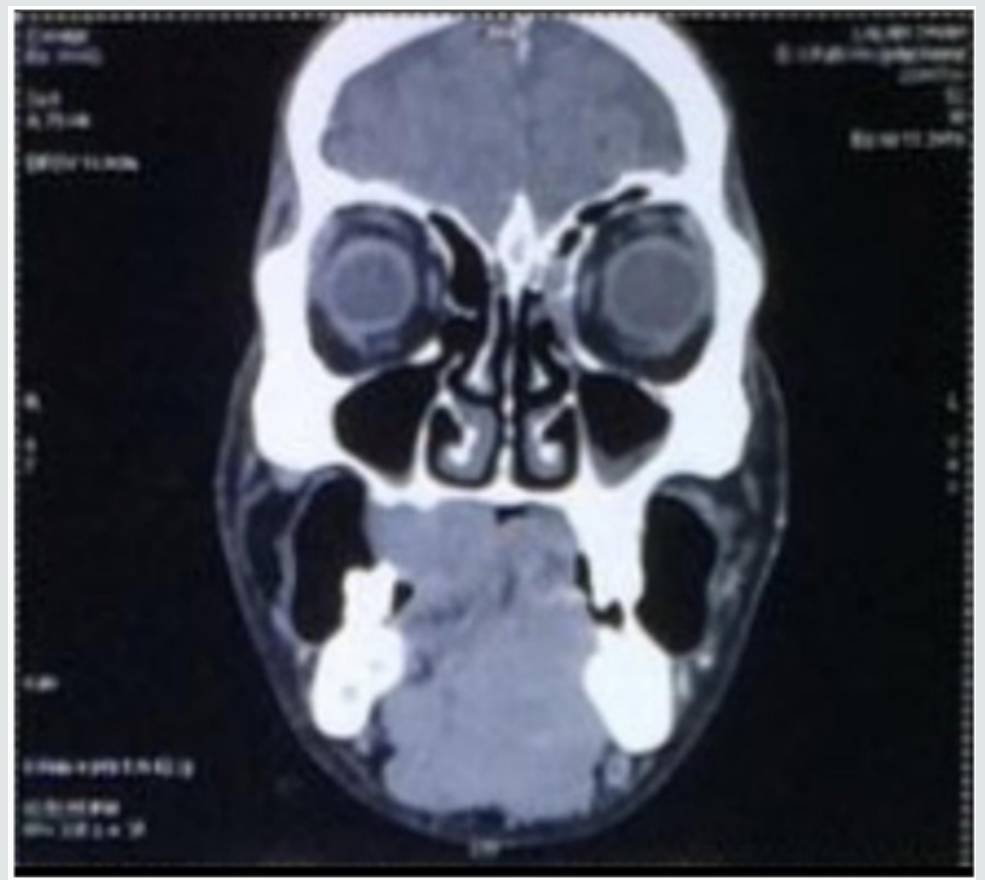

Figure 2.

The samples were also subjected to Immunohistochemistry for classification, subtyping and further management. FNAC of the cervical lymph node showed similar morphology as that of the palatal swelling. Bone marrow aspirate had a hypercellular marrow with 25\% lymphocytes. IHC Markers used were CD20(clone L26), CD3(CLONE PS1), CD5(CLONE SP19), CD43(CLONE DF-P1), CD10(CLONE 56C6), Bcl2(CLONE 100/B5), Bcl6(CLONE-PG- B6P), Ki67 which reported as: IHC markers that turned out to be positive were - CD20(CLONE L26)- Positive; CD10- focal positive; Bcl2positive; Bcl2- positive; Bcl6- positive; Ki67-15-20\% in neoplastic follicles. IHC markers that were negative are - CD3- negative; CD5negative; CD43- negative; He was thus diagnosed as a case of NonHodgkin - Follicular lymphoma grade II. The patient was referred to the Department of Medicine Oncology for further management.

\section{Discussion}

Lymphomas are primary tumour of lymph nodes. They have been majorly divided as Hodgkin lymphomas and Non-Hodgkin lymphoma. Named after Dr Thomas Hodgkin who identified the cells for the first time. NHL is further classified as B cell or T cell lymphomas depending on the cells they affect. GI tract is the most common extra nodal site for NHL followed by Oral cavity. Within oral cavity, Waldeyer's ring is the most common area to be affected, other including mandible, hard palate, nasopharynx, parotid gland, paranasal sinuses, thyroid gland and orbit [1]. Incidence of oral cancers is high due to addiction to tobacco. Presentation of NHL with primary site in oral cavity is rare. The mean age of presentation of NHL is 42 years of age. and its incidence increasing as the age advances. They are ranked fifth in terms of cancer incidence and mortality worldwide [2]. Majority Non-Hodgkin lymphomas are that of B cell origin type. Presentation of the lymphomas in the oral cavity are usually tooth ache, numbness, painless swelling [3] The differential diagnosis for such swelling can be that of an infective aetiology, hence all baseline investigations like a complete blood count is equally mandatory. The presenting symptoms for lymphomas are an unexplained, painless swelling of the lymph node, , gradually increasing in size either in the neck, axilla or the groin region. It can also be associated with B symptoms (symptoms whose, presence or absence has an impact on outcome of disease) such as fever, night sweats, abdominal pain, unexplained weight loss.

Majority of the NHL's are B cell in origin. In our patient the immunohistochemistry was CD20 (Pan B cell marker) positive, CD3 
negative(Pan T cell marker) suggestive of Follicular lymphoma. The aetiology of Non-Hodgkin lymphoma remains unknown. It shows a strong association with immunocompromised patients [4]. In our patient there was no evidence of any immunocompromised state, His serological status was Negative for HIV. Lifestyle factors like smoking, tobacco chewing have all direct adverse effects with NonHodgkin lymphomas. There is a twofold risk for NHL in cigarette smokers and three-fold in bidi smokers [3] Standard treatment modalities for NHL is chemotherapy. Generally, a combination of Chemotherapy (cyclophosphamide, doxorubicin, vincristine, prednisone) and radiation is recommended [5].

\section{Conclusion}

Non-Hodgkin lymphomas of hard palate are a rare entity Clinicians attending patients who come with growth in the oral cavity, or over the hard palate must rule out NHL as their differential diagnosis [6,7]. When detected in time, at early stages and treated either surgically or by chemotherapy, the prognosis of such patients is good [8].

\section{References}

1. Paes FM, Kalkanis DG, Sideras PA, Serafini AN (2010) FDG PET/CT of extranodal involvement in non-Hodgkin lymphoma and Hodgkin disease. Radiographics 30: 269-291.
2. Even Sapir E, Lievshitz G, Perry C, Herishanu Y, Lerman H, et al. (2007) Fluorine-18 fluorodeoxyglucose PET/CT patterns of extranodal involvement in patients with Non- Hodgkin lymphoma and Hodgkin's disease. Radiol Clin N Am 45: 697-709.

3. Landis SH, Murray T, Bolden S, Wingo PA (1998) Cancer statistics 1998. CA Cancer J Clin 48: 6-29.

4. Angiero F, Stefani M, Crippa R (2006) Primary non-Hodgkin's lymphoma of the mandibular gingiva with maxillary gingival recurrence. Oral Oncol Extra 42:123-128.

5. Adami J, Nyren O, Bergstrom R, Ekbom A, Engholm G, Englund A, et al. (1998) Smoking and risk of leukemia, lymphoma and multiple myeloma. Cancer Causes Control 9: 49-56.

6. Sathiya M, Muthuchelian K (2009) Significance of immunologic markers in the diagnosis of lymphoma. Acad J Cancer Res 2: 40-50.

7. Vinoth PN, Selvan SM, Sahni L, Krishnaratnam K, Rajendiran S, et al. (2012) Primary extra nodal non-Hodgkin's lymphoma of the oral cavity in a young girl. Natl J Maxillofac Surg 3: 187-189.

8. Ramani P, Shaheen A, Janaki VR (2004) Primary extranodal nonHodgkin's lymphoma of the oral cavity. Indian journal of dermatology, venereology and leprology $70(3)$.

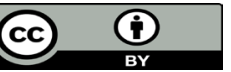

This work is licensed under Creative Commons Attribution 4.0 License

To Submit Your Article Click Here: Submit Article

DOI: $10.32474 /$ SJO.2019.03.000163

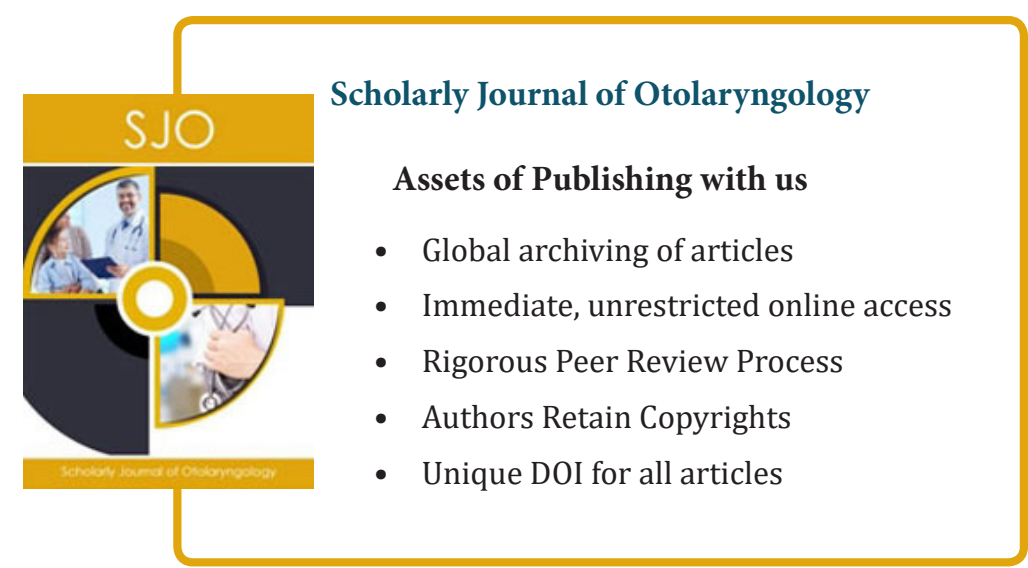

